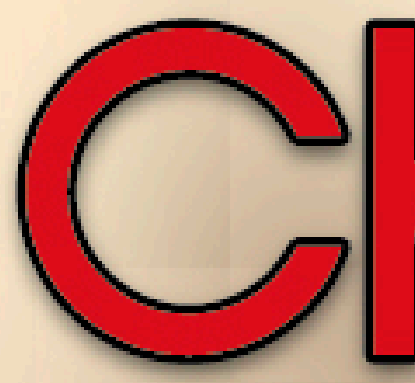

Revista Lattinoamericana de Comurnicación D
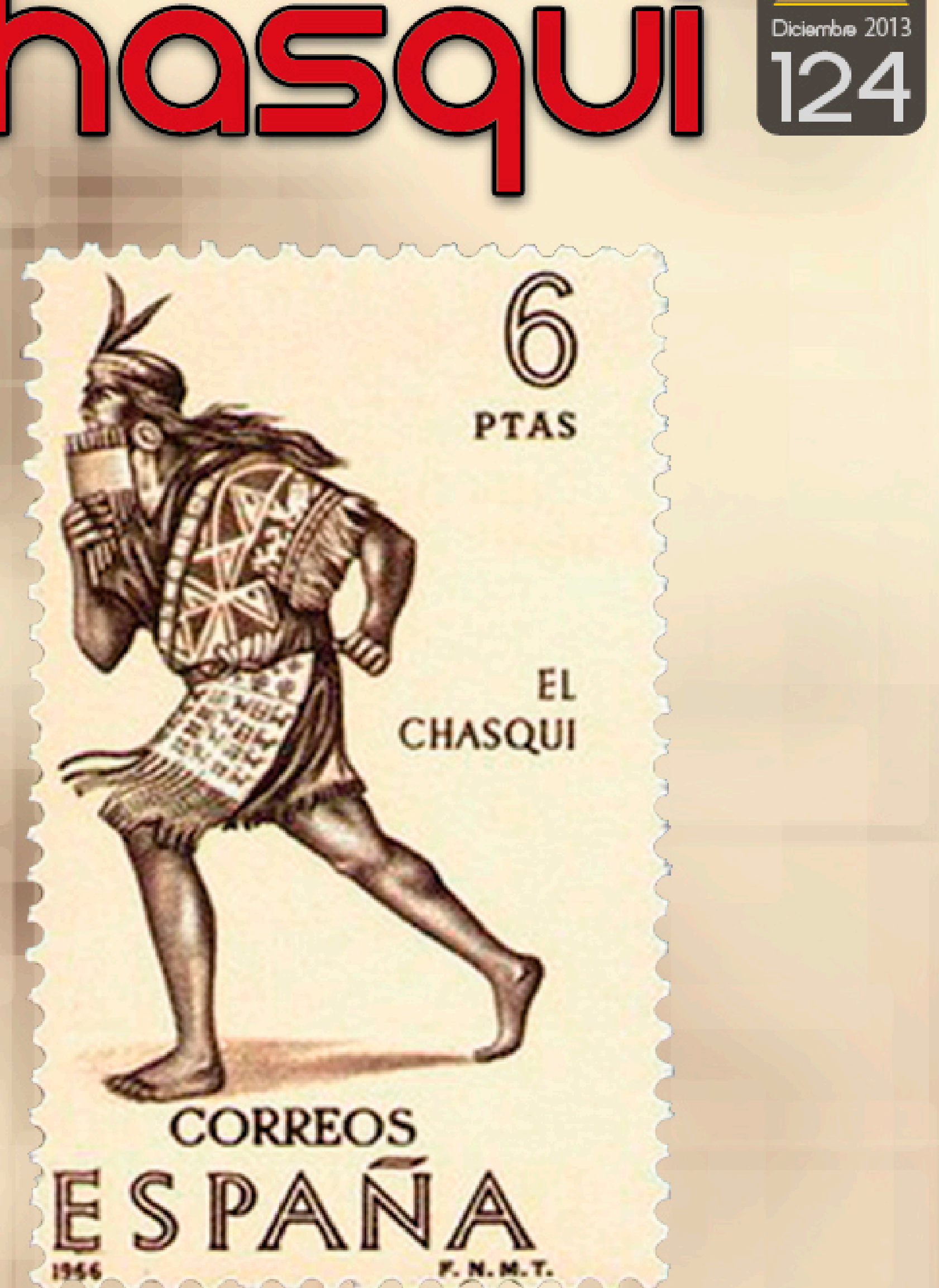

41 años $\cdot 2041$ artículos $\cdot 1332$ autores $\cdot 52$ países.

El rol social de la educación en medios 


\section{En esta edición}

\section{Tema central}

\section{El rol social de la educación en medios}

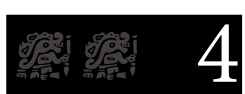

La urgencia de la competencia mediática en los programas escolares Alberto Parola Agueda Delgado Ponce

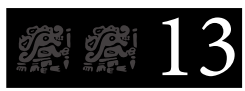

El desarrollo de la competencia mediática en personas mayores: una brecha pendiente Ángel Hernando Alejandra Phillippi

Presente y futuro de la alfabetización mediática en Europa:

el caso español y portugués Cristina Ponte

Paloma Contreras-Pulido

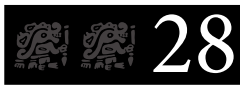

Avances para el desarrollo de la competencia mediática a partir del currículum de primaria en Colombia y España M. Amor Pérez-Rodríguez Yamile Sandoval

Las interacciones mediáticas de la juventud boliviana: la competencia mediática de jóvenes de La Paz

Vicent Gozálvez Pérez René Zeballos Clavijo

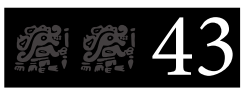

La competencia mediática en niños y jóvenes: La visión de España y Ecuador Isidro Marín-Gutiérrez Elena Díaz-Pareja Ignacio Aguaded

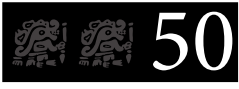

Competencia mediática ante la Agenda Setting: parámetros de actuación a nivel educativo Rosa García-Ruiz Diana Rivera-Rogel

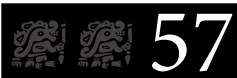

La alfabetización mediática en la televisión infantil online: programas del Canal Pakapaka Jacqueline Sánchez-Carrero Heleny Méndiz-Rojas

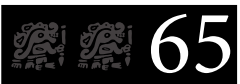

Educomunicación y radios universitarias: Panorama internacional y perspectivas futuras Ignacio Aguaded Daniel Martín-Pena

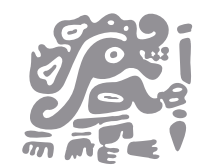




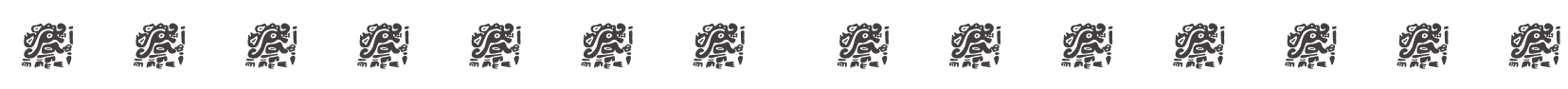

\section{Ensayo}

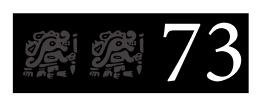

El sistema de comunicación en la Ley Orgánica de Comunicación del Ecuador: Un análisis desde el enfoque de las teorías de sociedad y masas de McQuail. Caroline Ávila

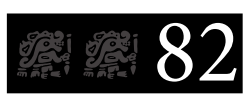

Periodismo y nuevas tecnologías: los ejes del pensamiento hegemónico y una posible contra agenda

Florencia Saintout

Redes de actores en medios de prensa.

Una metodología para abordar la hegemonía en los medios de comunicación: el ejemplo de las elecciones de 2013 en Paraguay. Rubén Juste de Ancos

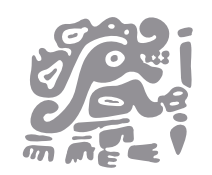

\section{Informe}

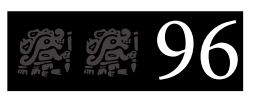

La colonización de la mirada. Un análisis de la información internacional en la prensa puertorriqueña Israel Rodríguez Sánchez

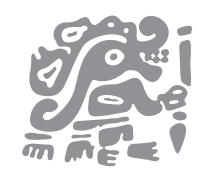

\section{Visual}

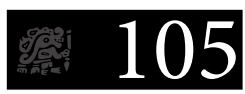

Miceláneos al fin del 2013

Diego S Acevedo

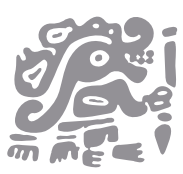

\section{Reseñas}

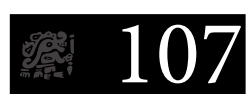

Anuario Obitel 2013

Memoria social y ficción televisiva en Iberoamérica

Alexandra Ayala Marín

Chasqui es un espacio para el desarrollo y difusión del pensamiento crítico en torno a la relación comunicación, cultura y política en el ámbito latinoamericano. Participan académicos, escritores, periodistas, artistas y otros pensadores unidos por la necesidad común de proponer, desde la comunicación, ideas impugnadoras al pensamiento dominante y al poder. 
Estamos inmersos en una sociedad mediatizada, donde la mayor parte de la información que nos llega, así como las comunicaciones, surgen a través de los medios de comunicación y las tecnologías, lo que demanda personas competentes en el uso eficaz y seguro de los medios, haciéndose indispensable una educación, no sólo desde la recepción, desde la decodificación sino a través de la creación, del manejo de los distintos lenguajes y del análisis crítico. Se genera por tanto una necesidad educativa que debería estar presente en las modificaciones curriculares. Dado que para incorporarse efectivamente en la sociedad actual no solo es necesaria una alfabetización lecto-escritora, como se ha venido entendiendo tradicionalmente, sino desde la atención, el conocimiento y utilización de las nuevas formas simbólicas mediante las que se transmiten las visiones del mundo.

Haciéndose eco de esta necesidad tan apremiante de educar en medios, entre las medidas políticas clave en el ámbito europeo está la introducción de una asignatura de «Educación mediática» en los colegios, que implica la capacidad de comprender y valorar críticamente los diversos aspectos de los distintos medios de comunicación, consiguiendo filtrar certeramente la información recibida a través del torrente de datos e imágenes. Esta asignatura, además, debe contar con un carácter práctico y multidisciplinar.

En esta línea, en 2009, la Comisión Europea presenta la «Recomendación sobre la alfabetización mediática», en la cual se anima a todos los países de la UE y a la industria de los medios de comunicación a abrir un debate en torno a la inclusión de la alfabetización mediática en el plan de estudios obligatorio y como parte de la oferta de competencias clave para el aprendizaje permanente. Con el fin de facilitar este debate, la Comisión creó en 2011 un grupo de expertos centrado en la alfabetización mediática en las escuelas cuyo objetivo no es otro que examinar el lugar actual de la alfabetización mediática en las escuelas de todos los países representados y debatir su posible inclusión en la educación formal.

A pesar de estas indicaciones, y de la urgencia de educar en medios, son aún muchos los pasos que hay que dar para que la competencia mediática se integre de una manera eficaz en los programas escolares. No obstante, en las siguientes líneas vamos a comprobar si tanto España como Italia se encuentran ya en el camino adecuado.

\section{La competencia mediática en los programas escolares. El caso español}

En el marco general, la LOE (Ley Orgánica de Educación 2/2006, de 3 de mayo) recoge la educación mediática entre los principios pedagógicos de las distintas etapas educativas. De este modo, «En ambos ciclos de la educación infantil se atenderá progresivamente [...] a las manifestaciones de la comunicación y del lenguaje...» (Art. 14.3). Y para Educación Primaria y Secundaria, «Sin perjuicio de su tratamiento específico en algunas de las áreas de la etapa, la comprensión lectora, la expresión oral y escrita, la comunicación audiovisual, las tecnologías de la información y la comunicación y la educación en valores se trabajarán en todas las áreas» (Art. 19.2; 24.7 y 25.5). Principios estos que se concretan en los decretos de enseñanzas mínimas que regulan los currículos de las diferentes etapas, a través de los objetivos y contenidos de las distintas áreas y de la definición de las Competencias Básicas.

En lo que se refiere al tratamiento de la educación mediática desde las distintas áreas, se observa una atención irregular, no apareciendo en todas las materias ni en la misma proporción objetivos, contenidos y criterios de evaluación relacionados con la educación en medios de comunicación y tecnologías.

Enlaetapadeeducacióninfantil,además deatender al desarrollo afectivo, al movimiento, a los hábitos de control corporal, las pautas de convivencia y el descubrimiento de las características sociales y físicas del medio, se presta atención a las manifestaciones tanto de la comunicación como del lenguaje y la relación social, ámbitos en los que los medios de comunicación y las tecnologías tienen un papel especial. De este modo, en el segundo ciclo de infantil, se atenderá a la importancia de las tecnologías como parte del entorno y la importancia que éstas tienen en sus vidas y, específicamente, en el área de Lenguaje: comunicación y representación, se le concede una especial relevancia a iniciar a los niños en la comprensión de los mensajes audiovisuales y su adecuada utilización puesto que son un aspecto que está muy presente en sus vidas diarias y necesitan de una formación específica como lenguaje y herramienta comunicativa. 
Para la etapa de educación primaria, se define entre sus objetivos generales el de «Iniciarse en la utilización, para el aprendizaje, de las tecnologías de la información y la comunicación desarrollando un espíritu crítico ante los mensajes que reciben y elaboran». En cuanto a las distintas áreas, entre sus objetivos y contenidos se incluyen conceptos presentes en la competencia mediática, aunque no en la misma proporción ni atendiendo a todos los aspectos de ésta. De este modo, la utilización de las tecnologías y los medios de comunicación para la búsqueda, selección y valoración crítica de la información está presente en la mayoría de las materias, mientras que el conocimiento de los distintos códigos y la creación de textos mediáticos aparecen más específicamente en el área de lengua castellana y literatura o expresión artística. Por su parte, en la educación secundaria, como etapa final de la educación obligatoria, se requiere de una profundización y consolidación de los aspectos trabajados en la etapa anterior. Entre sus objetivos generales se concreta la utilización de fuentes de información y la preparación en el campo de las TIC, dimensiones que se trabajan en todas las áreas, concretamente, el uso de los medios de comunicación y las tecnologías como fuentes de información, en la que el alumnado debe ser capaz de comprender, interpretar y valorar críticamente la información hallada. En cuanto al resto de dimensiones relacionadas con la competencia mediática, aunque se hallan de manera transversal en las distintas áreas, el tratamiento es parcial y segmentado, dificultando una visión completa y adecuada que desemboque en la adquisición de dicha competencia. Así, encontramos que los alumnos se enfrentan a la comunicación en las áreas de lengua (castellana y extranjera) sin una atención específica a las características propias de las nuevas formas de comunicación que trae consigo el nuevo ecosistema comunicativo. Y los distintos códigos y lenguajes, visual y musical, se trabajan de manera específica en las áreas de educación plástica y visual y educación musical, respectivamente. Quedando, finalmente, desatendidos aspectos como la atención a la emoción, a la influencia de los medios y la participación ciudadana, tan importantes para la incorporación efectiva de los jóvenes en la sociedad actual.

Además de los objetivos, contenidos y criterios de evaluación presentes en las distintas áreas, o más bien, relacionadas con estos, en el Anexo I de los Reales Decretos, tanto de primaria como de secundaria, se incluyen ocho competencias que difieren en parte de las aportadas por el marco de referencia. De entre éstas, el Tratamiento de la información y competencia digital sería la que más atañe al desarrollo de la educación mediática, en tanto se define como «disponer de habilidades para buscar, obtener, procesar y comunicar información, y para transformarla en conocimiento. Incorpora diferentes habilidades, que van desde el acceso a la información hasta su transmisión en distintos soportes una vez tratada, incluyendo la utilización de las tecnologías de la información y la comunicación como elemento esencial para informarse, aprender y comunicarse». Donde el núcleo de la alfabetización mediática estaría según Aparici y otros (2010: 20), en «el dominio de lenguajes específicos básicos (textual, numérico, icónico, visual, gráfico y sonoro) y de sus pautas de decodificación y transferencia, así como aplicar en distintas situaciones y contextos el conocimiento de los diferentes tipos de información, sus fuentes, sus posibilidades y su localización, así como los lenguajes y soportes más frecuentes en los que ésta suele expresarse», en definitiva, el dominio de los diferentes lenguajes y soportes y su aplicación a la comprensión, la interpretación crítica, la comunicación y la expresión. Dejando de lado dimensiones e indicadores necesarios para la adquisición de la competencia mediática si atendemos a los trabajos de Ferrés y Piscitelli (2012), Celot y Pérez Tornero (2009) o Pérez Rodríguez y Delgado Ponce (2012).

\section{La competencia mediática en los programas escolares. El caso italiano}

Con respecto a las nuevas Indicaciones nacionales para el currículo de la escuela de la infancia y del primer ciclo de educación ${ }^{7}$ (de ahora en adelante Indicaciones), en Italia, podemos ver, en general, los conceptos de meta para el desarrollo de competencias y de certificación de las competencias: en este sentido, se presta una atención particular a cómo el estudiante moviliza, controla y organiza sus propios recursos (conocimiento, habilidad, actitudes, emociones) para responder eficazmente a las situaciones que la realidad ofrece, en relación a sus potencialidades y aptitudes. Asimismo, surge la necesidad de un desarrollo para el conjunto del primer ciclo de la educación (de 3 a 14 años) y luego continuar su consolidación, en vista de metas siempre nuevas

1 Ver www.indicazioninazionali.it. 
y diversificadas, hasta el final de la educación secundaria. Con respecto al perfil de competencias para el final del primer ciclo de la educación, se establece que el estudiante debe poseer «buenas habilidades digitales, utilizar con conciencia las nuevas tecnologías de la comunicación para investigar y analizar datos e informaciones, para distinguir las informaciones fiables de las que requieren mayor profundización, seguimiento y verificación y para interactuar con diferentes temas en el mundo», podemos vislumbrar el pequeño paso dado por las nuevas Indicaciones en la dirección de una formación siempre más «media educativa», incluso si pensamos que este paso podría y debería ser más decisivo y eficaz. Según el documento, las metas constituyen los criterios para la evaluación de las competencias previstas, mientras que los objetivos de aprendizaje identifican los campos del saber, conocimiento y habilidad, considerados indispensables para alcanzar las metas establecidas para cada disciplina. Importantes son los conceptos de comunidad educativa, comunidad profesional y ciudadanía (también digital, añadimos), aplicables ya a partir de la escuela infantil. Desde los 3 años (con las temáticas relativas a sí mismo y al otro, al cuerpo y el movimientos, a las imágenes, sonidos y colores, a los discursos y las palabras y al conocimientos del mundo), los niños exploran, en la escuela, la potencialidad ofrecida por la tecnología mientras, de los 6 años en adelante, en la primaria y la secundaria de primer grado, continúan desarrollando su competencia, siempre de forma más amplia y transversal, en las disciplinas ${ }^{2}$ específicas. Gradualmente,

2 Nos referimos a: lengua italiana, segunda lengua, historia, geografía, matemáticas, ciencia, música, arte e imagen, educación física y tecnología. En síntesis: En relación a la lengua italiana se habla de producción de textos multimedia y la consulta de diccionarios y repositorios tradicionales y online; lengue comunitaria: uso de tecnología informática y de programas radiofónicos y televisivos; historia: relación entre los recursos digitales y las fuentes históricas: geografia: fotografías actuales y de época, sistemas informativos geográficos para comunicar eficazmente informaciones espaciales, cartografía computerizada y programas multimedia de visualización elevada, matemáticas: hoja de cálculo; música: instrumentos electrónicos, danza, teatro artes visuales y multimedia y software específicos para la elaboración sonora y musical. En cuanto a la tecnología, se trata de un estudiante que expresa una actitud crítica y una mayor conciencia de los efectos sociales y culturales de su distribución, a las consecuencias relacionales y psicológicos de las posibles formas de uso, al impacto del medio ambiente o la salud, tarea educativa fundamental que será compartida entre las diferentes disciplinas y proyecta y realiza una representación gráfica o infográfica [...] utilizando elementos del diseño técnico u otros lenguajes multimedia a través de entornos digitales, es preciso, posteriormente, hacer consciente al alumno de su propio estilo de aprendizaje (nivel metacognitivo), un aspecto extraordinariamente importante para el desarrollo sólido de competencias, las cuales se deberán poder someter, con cierta regularidad, a un proceso de autoevaluación. En relación a los «espacios disponibles» en la escuela del primer ciclo, se denota que el estudiante encuentra estímulos para investigar el pensamiento analítico y crítico, aprende a aprender, cultiva la fantasía y el pensamiento original, compara para buscar significados y compartir esquemas decomprensión dela realidad, elemento fundamental, como se verá, para configurar una competencia mediática, especialmente aquélla relacionada con la autonomía crítica (critical thinking skill). Otras claves interesantes se refieren a la mejora de la experiencia, a la atención a la diversidad y la inclusión, la motivación para fomentar la exploración y el descubrimiento predominante en un taller didáctico, el estímulo del aprendizaje colaborativo, ser consciente de su propio modo de aprender y el avance para adquirir un estilo de aprendizaje propio, todos los ingredientes que van a componer un sistema de conceptos que contribuyen al desarrollo del conocimiento y la habilidad y la meta-calidad muy «indicadora» (en el sentido de indicadores) de una competencia mediática.

Entre todas las disciplinas indicadas en la nota 2, la materia arte e imagen muestra los espacios más interesantes: específicamente, los elementos que parecen más relacionados con la educación mediática (de ahora en adelante EM), porque nos centramos en el hecho de que el estudiante va a reconocer, valorar y ordenar el conocimiento 
y la experiencia obtenida en el campo expresivo y multimedia también fuera de la escuela, como elementos útiles en el proceso de formación de la capacidad de reflexión crítica. En tal sentido, se podrá/deberá entonces realizar trabajos personales y creativos eligiendo funcionalmente las diferentes técnicas y materiales también con la integración de más medios y códigos expresivos, dominando los elementos principales del lenguaje visual. Las materias ciencias y educación física no ofrecen, por otra parte e increíblemente, sugerencias significativas.

En sustancia, dentro de las Indicaciones nacionales encontramos una mayor convicción en la identificación de la carretera que conduce a la educación en medios en las escuelas, sin embargo, no tanto como una disciplina, sino como un enfoque que permite la conexión entre diferentes disciplinas y como un método de enseñanza. Probablemente, se podrá hacer más desde el punto de vista de los ejemplos y del uso del lenguaje: en cuanto al lado positivo, en algunas materias es posible rastrear las metas y objetivos de aprendizaje relacionados con la EM e, hilado fino, una posible referencia no explícita a la competencia mediática; mientras en relación a los aspectos negativos, los medios no representan casi nunca el centro de interés ni como instrumentos funcionales para la enseñanza ni como objeto de estudio y de expresión.

Podemos sostener en este punto que se ha realizado un esfuerzo en la dirección hacia una educación de los medios más presente y estructurada, con el conocimiento de que aún es necesario recoger las buenas prácticas, poner en práctica una amplia investigación y confiar en la profesionalidad y la creatividad de los docentes.

\section{Acciones para el desarrollo de la competencia mediática en los programas escolares}

A la luz de cuanto se ha dicho, estamos ahora en situación de proponer algunas sugerencias para el desarrollo y la valoración de las competencias digitales, y más específicamente mediáticas en la escuela.

Con todo lo dicho, creemos que un primer paso ineludible en este proceso de educación en medios sigue siendo la evaluación de carencias y necesidades a este respecto, que determine unas pautas eficaces de intervención. De ahí, sin olvidar otros trabajos sobre evaluación (Celop y PérezTornero, 2009) o partiendo de investigaciones precedentes en este sentido (Aguaded, 2011; Ferrés, 2011), en España, dentro del ámbito nacional, enmarcado en la convocatoria I+D del Ministerio de economía y competitividad, se está llevando a cabo el proyecto coordinado «La competencia mediática en un entorno digital: Diagnóstico de necesidades en tres ámbitos sociales» (www.competenciamediatica.es). Dentro del cual se sitúa el subproyecto titulado «La enseñanza obligatoria ante la competencia en comunicación audiovisual en un entorno digital», que realiza, entre otras tareas, el análisis de los planes de estudio y la evaluación de la competencia mediática en la educación infantil, primaria y secundaria, con la finalidad de determinar las acciones educativas necesarias. Para ello se siguen las dimensiones propuestas por Ferrés $(2007,2012)$ que se centran en dos ámbitos: el de la expresión y el de la interpretación, y que se consideran imprescindibles para la adquisición de la competencia mediática: lenguajes (conocimiento de los códigos que hacen posibles los diversos lenguajes, de los discursos o textos en los que cristalizan y de los géneros y formatos discursivos, así como la capacidad de expresarse con una mínima corrección mediante los distintos sistemas de representación), tecnología (conocimiento de las herramientas y de los entornos que hacen posible una comunicación multimedial y multimodal y capacidad de manejar estas herramientas de manera autónoma), procesos de interacción (capacidad de dilucidar por qué nos gustan unos productos, individual y socialmente, y cómo ejerce su influencia y capacidad de interaccionar con las pantallas y con otras personas $y$ colectivos), procesos de producción y difusión (conocimiento de los factores que convierten las producciones corporativas en mensajes sometidos a los condicionamientos de la industria mediática y capacidad de trabajar de manera colaborativa en la producción de mensajes y capacidad de difundirlos), ideología y valores (capacidad de analizar y evaluar críticamente los mensajes audiovisuales y capacidad de producir mensajes que transmitan valores y contribuyan a la mejora del entorno social) y la dimensión estética (capacidad de analizar y valorar los mensajes audiovisuales desde el sentido estético y capacidad para relacionarlos con otras formas de manifestación mediática y artística). 
MED (Asociación italiana para la educación de los medios y la comunicación) lleva muchos años realizando un trabajo de formación e investigación sobre el campo en esta dirección y ha publicado numerosos volúmenes sobre los temas aquí indicados. Especialmente, desde el año 2006, ha formulado un marco teórico común que comprende cinco competencias mediáticas para desarrollar desde la educación infantil hasta la educación secundaria de segundo grado: competencia de lectura (que sitúa sustancialmente la capacidad de análisis y que responde a la pregunta «¿cuáles son los elementos lingüísticos que caracterizan estos mensajes?»), de escritura (que se centra principalmente sobre la producción creativa responsable con los lenguajes digitales y mediáticos y que responde a la pregunta «ipor qué el autor ha escrito este mensaje?» y « ¿soy capaz de escribir y producir mensajes mediáticos?»), crítica (que permite a los niños y jóvenes disponer de un punto de vista autónomo, poner de relieve los valores comunes y ser capaz de distinguir los diversos niveles de los mensajes y la intencionalidad del emisor, y que responde a la pregunta "¿desde qué punto de vista y desde qué valores se inspira este mensaje?»), de recepción (fundamentalmente basada en la educación de la organización cotidiana del uso combinado de los diferentes medios, la autodeterminación y la selección y que responde a la pregunta "¿estoy verdaderamente interesado en recibir este mensaje?» y «ipuedo tomar decisiones conscientes sobre la recepción?») y, finalmente, de ciudadanía (que comprende las precedentes y que viene desarrollada durante todo el currículo escolar y, después, a lo largo de toda la vida y que responde a la pregunta "¿qué efecto tiene este mensaje en la sociedad?»).

Este modelo teórico nos ha permitido crear diferentes rutas, para realizar observaciones e investigaciones en el campo y para construir modelos y herramientas de verificación, a partir de la evaluación del aprendizaje (Kirkpatrick, 1994) con los niveles de satisfacción, aprendizaje y cambio, y elaborar un sistema de indicadores para la evaluación de la competencia mediática basado en el modelo RIZA³.

A continuación, como ejemplo, presentamos un posible perfil de competencia general para el área

\footnotetext{
3 Modelo teorizado de R. Trinchero y presente en Media, linguaggi, creatività, op.cit., pp. 51-68, sobre la base de los estudios de Le Boterf (2000).
}

crítico mediática. En cuanto a los recursos (es decir los saberes), el niño debe ser capaz de comprender los conceptos relacionados con la dimensión ética (los valores de fondo, las opciones morales, principios éticos) estética (rasgos estilísticos del género y del autor), y socio-cultural (antecedentes mentales, inscripciones históricas, el mundo de las ideas, perspectivas culturales); en relación a las denominadas estructuras de interpretación (relacionadas con la capacidad de saber «leer» una situación) el estudiante debe saber reconocer los significados que un mensaje lleva implícitamente, los puntos de vista y los valores subyacentes de los mensajes, el fondo del mensaje y los rasgos éticos, estilísticos y culturales de un texto mediático y, por otra parte, saber interpretar las coordenadas de sentido que inspiran los textos mediáticos; referente a la estructura de acción, debe ser capaz de llevar a cabo la perspectiva presente en el texto y evaluar un mensaje en los puntos de vista y los valores que encarna $y$, finalmente, con respecto a las estructuras de autorregulación, saber reflexionar sobre la propia crítica de los productos mediáticos e identificar, de manera guiada, los propios errores. Naturalmente, el mismo trabajo se puede aplicar, con diferentes conceptos, a las otras competencias mediáticas. Este modelo, complejo en su formulación y, al mismo tiempo, fácil de aplicar permite al educador construir instrumentos de evaluación como cuestionarios y rúbricas, pero también las situaciones reales en las que el estudiante crea actuaciones que le permiten demostrar su capacidad para recordar los conocimientos, interpretar una situación desde diferentes puntos de vista, ejecutar participación observable y verbalizar los modos de autoregulación en la lectura, la escritura, la crítica y el uso de los mensajes mediáticos.

La transición desde un marco teórico, a una clara estrategia de acción $y$, finalmente, a una propuesta valorativa basada en indicadores de competencias, resulta fundamental para la certificación de una competencia que prevé tres niveles ${ }^{4}:$ base (el estudiante realiza tareas sencillas en situaciones conocidas, mostrando que posee los conocimientos y habilidades esenciales y es capaz de aplicar las normas y procedimientos básicos), intermedio (el estudiante realiza tareas y resuelve problemas complejos en situaciones conocidas, toma decisiones, mostrando ser capaz de utilizar el conocimiento y la habilidad

4 En este sentido, véase la Orden Ministerial 9 del 27 de enero 2010 en el sitio www.edscuola.eu. 
adquirida) y avanzado (el estudiante realiza tareas y problemas complejos en situaciones también desconocidas, mostrando dominio en la utilización del conocimiento y la habilidad y sabe cómo proponer y apoyar sus propias opiniones y asumir autónomamente las decisiones). Es importante prestar atención a los dos discriminantes: la complejidad de la tarea y la situación nueva: quien es competente, o ha alcanzado un nivel avanzado de competencia, se ocupa de ambos aspectos y domina totalmente el procedimiento (de hecho, los procedimientos) para la solución de la tarea. Sin embargo, la única posibilidad de certificar las competencias mediáticas (o mejor, digitales por aquello que se dice en las Indicaciones) es el eje de los lenguajes (que se expresa en la cuarta competencia clave, la digital, de las propuestas por el Parlamento Europeo en julio de 2006). En realidad nos gustaría que este eje fuese transversal, ya que puede intervenir en todas las disciplinas, reforzándolas desde el punto de vista de la innovación didáctica, de la mejora del aprendizaje y del desarrollo de la competencia mediática "crítica» (y de las otras competencias del modelo teórico propuesto) porque, finalmente, se trata de construir la capacidad para «leer» y «escribir» y saber elegir en la dirección de una ciudadanía siempre activa, participativa, democrática y libre.

\section{Conclusiones}

Como hemos visto, podemos decir que la educación tiene aún una asignatura pendiente en la adaptación a las nuevas realidades y la atención a las necesidades formativas de alumnos, profesores y familias. La rápida y continua transformación social y los avances tecnológicos, dificultan notablemente la adopción de una competencia mediática de manera integrada por parte del sistema educativo, que sigue aún anclado en la época industrial y en el formato papel. Se requieren por tanto cambios de fondo para que la educación formal acabe por adaptarse a este ecosistema mediático. Afortunadamente, y en la mayoría de los casos en contacto con estos entornos formales, podemos encontrar la labor de grupos, asociaciones, investigadores... que creen en la urgencia de educar en medios y afrontan trabajos de investigación, acciones formativas, publicaciones, etc. a este respecto. Impulsando, de este modo, que los ciudadanos en general y los niños y jóvenes en particular sean capaces de leer y escribir, de informarse y comunicarse eficazmente con y a través de los medios y las tecnologías mostrando una actitud crítica y participativa; en definitiva, ser competentes mediáticos. 翟

\section{Bibliografía}

Aguaded, J. Ignacio (2011). El grado de competencia mediática en la ciudadanía andaluza. Huelva: Grupo Comunicar Ediciones, Universidad de Huelva.

Aparici, Roberto y otros (2010), La educación mediática en la Escuela 2.0. (http://ntic.educacion.es/w3/ web_20/informes/educacion_mediatica_e20_ julio20010.pdf) (Consulta: 14-11-2012).

Celot, Paulo (Project Coordinator and editor) y PérezTornero, Jose-Manuel (Scientific Coordinator) (2009). Study on Assessment Criteria for Media Literacy Levels - A comprehensive view of the concept of media literacy and an Understanding of how media literacy level in Europe Should Be Assessed. Brussels: European Commission. (http://ec.europa.eu/culture/media/literacy/docs/ studies/eavi_study_assess_crit_media_lit_levels_ europe_finrep.pdf) (Consulta: 26-05-2013).

Ferrés, Joan y Piscitelli, Alejandro (2012). La competencia mediática: propuesta articulada de dimensiones e indicadores. Comunicar, 38, 75-82.

Ferrés, Joan, García-Matilla, Agustín; Aguaded, J. Ignacio y otros (Coord.) (2011). Competencia mediática. Investigación sobre El grado de competencia de la ciudadanía en España. Ministerio de Educación, Instituto de Tecnología Educativa.

Ferrés, Joan (2007). La competencia en comunicación audiovisual: propuesta articulada de dimensiones e indicadores. Quaderns del CAC, 25, 9-17.

Kirkpatrick, Donald (1994). Evaluating Training Programs. San Francisco: Berrett-Koehler Publishers, Inc.

Le Boterf, Guy (2000). Construire les compétences individuelles et collectives. Paris: Les Editions d'Organisation.

Parola Alberto y Ranieri, Maria (2010). Media Education in Action. A Research Study in Six European Country, Firenze, FUP.

Parola, Alberto, Rosa A. y Giannatelli Roberto (2012). Media, linguaggi, creatività. Trento: Erickson, 2012.

Pérez-Rodríguez, María-Amor y Delgado-Ponce, Águeda (2012). De la competencia digital y audiovisual a la competencia mediática: dimensiones e indicadores. Comunicar, 39, 25-34.

Trinchero, Roberto (2012). Costruire, valutare, certificare competenze. Proposte di attività per la scuola. Milano: Franco Angeli, 2012. 